\title{
On a class of weighted Gauss-type isoperimetric inequalities and applications to symmetrization
}

\author{
Michele Marini (*) - Berardo Ruffini (**)
}

ABSTRACT - We solve a class of weighted isoperimetric problems of the form

$$
\min \left\{\int_{\partial E} w e^{V} d x: \int_{E} e^{V} d x=\text { constant }\right\}
$$

where $w$ and $V$ are suitable functions on $\mathbb{R}^{d}$. As a consequence, we prove a comparison result for the solutions of degenerate elliptic equations.

Mathematics SubJect Classification (2010). 26D10, 35J15, 26D20.

KEYWORDs. Weighted isoperimetric inequalities, symmetrizations, rearrangements.

\section{Introduction}

In the celebrated paper [12], G. Talenti established several comparison results between the solutions of the Poisson equation with Dirichlet boundary condition (with suitable data $f$ and $E$ ):

$$
-\Delta u=f \text { in } E, \quad u=0 \text { on } \partial E
$$

and the solutions of the corresponding problem where $f$ and $E$ are replaced by their spherical rearrangements (see [10, Chapter 3] for the definition and main properties of spherical rearrangement). Precisely, he proves that if we denote by $v$ the solution of the problem with symmetrized data, then the

(*) Indirizzo dell'A.: Scuola Normale Superiore, Piazza dei Cavalieri 7, 56126 Pisa, Italy.

E-mail: michele.marini@sns.it

(**) Indirizzo dell'A.: Institut Fourier, 100 rue des maths, BP 74, 38402 St Martin d'Hères cedex, France.

E-mail: berardo.ruffini@ujf-grenoble.fr

The author was partially supported by the PRIN 2010-2011 "Calculus of Variations". 
rearrangement $u^{*}$ of the (unique) solution $u$ of (1.1) is pointwise bounded by $v$. Moreover he shows that the $L^{q}$ norm of $\nabla u$ is bounded, as well, by the $L^{q}$ norm of $\nabla v$, for $q \in(0,2]$. The proof of these facts basically relies on two ingredients: the Hardy-Littlewood-Sobolev inequality and the isoperimetric inequality (see [1] and [10] for comprehensive accounts on the subjects).

Later on, following such a scheme, many other works have been developed to prove analogous comparison results related to the solutions of PDEs involving different kind of operators, see for instance [2], [3], [6], [7], [8], [9], [13] and the references therein. A recurring idea in these works is, roughly speaking, the following: the operator considered is usually linked to a sort of weighted perimeter. Thus initially it is necessary to solve a corresponding isoperimetric problem; then the desired comparison results can be obtained following the ideas contained in [12].

For example in [3] the authors consider a class of weighted perimeters of the form

$$
P_{w}(E)=\int_{\partial E} w(|x|) d \mathcal{H}^{d-1}(x),
$$

where $E$ is a set with Lipschitz boundary and $w: \mathbb{R} \rightarrow[0, \infty)$ a non-negative function, and prove, under suitable convexity assumptions on the weight $w$, that the ball centered at the origin is the unique solution of the mixed isoperimetric problem

$$
\min \left\{P_{w}(E):|E|=\text { constant }\right\}
$$

where $|\cdot|$ denotes the $d$-dimensional Lebesgue measure. As a consequence they prove comparison results, analogous to those considered by Talenti in [12], for the solutions of

$$
-\operatorname{div}\left(w^{2} \nabla u\right)=f \text { in } E, \quad u=0 \text { on } \partial E .
$$

Recently in [4], L. Brasco, G. De Philippis and the second author proved a quantitative version of the weighted isoperimetric inequality considered in [2]. Their proof is achieved by means of a sort of calibration technique. One advantage of this technique is that it is adaptable to other kind of problems, as that of considering other kind of functions in the weighted perimeter (e.g. Wulff-type weights, see [5]), or that of considering different measured spaces, as $\mathbb{R}^{d}$ endowed with the Gauss measure.

In this paper we consider degenerate elliptic equations with Dirichlet boundary condition of the form

$$
-\operatorname{div}\left(w^{2} e^{V} \nabla u\right)=f e^{V} \text { in } E, \quad u=0 \text { on } \partial E
$$

where $w$ and $V$ are two given functions, and we aim to prove analogous 
comparison results as those in [12]. The particular form in which is written the measure $e^{V}$ is due to the later applications, whose main examples are Gauss-type measures, that is $V(x)=-c|x|^{2}$. Bearing in mind this instance, we consider a class of mixed isoperimetric problems of the form

$$
\min \left\{P_{w e^{V}}(E): \int_{E} e^{V}=\text { constant }\right\}
$$

and prove, by means of a calibration technique reminiscent of that developed in [4], that the solutions, under suitable assumptions on $V$ and $w$, are half-spaces, see Proposition 3.1 and Theorem 3.6. Then, using a suitable concept of rearrangement related to the measures considered, we prove, in the Main Theorem in Section 4, comparison results between the solutions of (1.2) and the solutions of the same equation with rearranged data.

\section{Preliminaries on rearrangement inequalities}

In this section we introduce the main definitions and properties about the concept of symmetrization and rearrangement we shall make use of.

Let $\mu$ be a finite Radon measure on $\mathbb{R}^{d}$, a right rearrangement with respect to $\mu$ is defined, for any Borel set $A$, as

$$
R_{A}^{\mu}=\left\{\left(x_{1}, x^{\prime}\right) \in \mathbb{R} \times \mathbb{R}^{d-1}: x_{1}>t_{A}\right\},
$$

where $t_{A}=\inf \left\{t: \mu(A)=\mu\left(\left\{\left(x_{1}, x^{\prime}\right) \in \mathbb{R} \times \mathbb{R}^{d-1}: x_{1}>t\right\}\right)\right\}$. Notice that if $d \mu=f d x$, for some positive and measurable function $f$, then the value of $t$ is uniquely determined.

Given a non-negative Borel function $f: \mathbb{R}^{d} \rightarrow[0,+\infty)$, we call right increasing rearrangement of $f$ the function $f^{* \mu}$ given by

$$
f^{* \mu}(x)=\int_{0}^{+\infty} \chi_{R_{\{f>t\}}^{\mu}}(x) d t
$$

where $\chi_{A}$ is the characteristic function of the set $A$. As an aside we notice that the right increasing rearrangement of the characteristic function of a Borel set $A$ coincides with the characteristic function of $R_{A}^{\mu}$. Clearly $f^{* \mu}$ is non-negative, increasing with respect to the first variable $x_{1}$, and constant on the sets $\left\{\left(x_{1}, x^{\prime}\right) \in \mathbb{R} \times \mathbb{R}^{d-1}: x_{1}=t\right\}$, for $t \in \mathbb{R}$. Moreover $f$ and $f^{* \mu}$ share the same distribution function:

$$
\mu_{f}(t):=\mu(\{f>t\})=\mu\left(\left\{f^{* \mu}>t\right\}\right)=\mu_{f^{* \mu}}(t) .
$$


We furthermore define $f^{\star \mu}: \mathbb{R}^{+} \rightarrow \mathbb{R}^{+}$as the smallest decreasing function satisfying $f^{\star \mu}\left(\mu_{f}(t)\right) \geq t$; in other words

$$
f^{\star \mu}(s)=\inf \left\{t>0: \mu_{f}(t)<s\right\} .
$$

It is useful to bear in mind that $\left\{s: f^{\star \mu}(s)>t\right\}=\left[0, \mu_{f}(t)\right]$ so that by the Layer-Cake Representation Theorem (see for instance [10]) we have

$$
\int_{0}^{\mu\left(\left\{x_{1}>t\right\}\right)} f^{\star \mu}(s) d s=\int_{t}^{\infty} \mu_{f}(s) d s=\int_{\left\{x_{1}>t\right\}} f^{* \mu}(x) d x .
$$

We conclude this section by proving the Hardy-Littlewood rearrangement inequality related to the right symmetrization.

LEMMA 2.1 (Hardy-Littlewood rearrangement inequality). Let $f$ and $g$ be non-negative Borel functions from $\mathbb{R}^{d}$ to $\mathbb{R}$. Then for any non-negative Borel measure $\mu$ we have

$$
\int_{\mathbb{R}^{d}} f g d \mu \leq \int_{\mathbb{R}^{d}} f^{* \mu} g^{* \mu} d \mu .
$$

Proof. We have

$$
\begin{aligned}
\int_{\mathbb{R}^{d}} f g d \mu & =\int_{\mathbb{R}^{d}} \int_{0}^{\infty} \int_{0}^{\infty} \chi_{\{f>t\}}(x) \chi_{\{g>s\}}(x) d t d s d \mu(x) \\
& =\int_{0}^{\infty} \int_{0}^{\infty} \int_{\mathbb{R}^{d}} \chi_{\{f>t\} \cap\{g>s\}}(x) d \mu(x) d t d s \\
& =\int_{0}^{\infty} \int_{0}^{\infty} \mu(\{f>t\} \cap\{g>s\}) d t d s \\
& \leq \int_{0}^{\infty} \int_{0}^{\infty} \min (\mu(\{f>t\}), \mu(\{g>s\})) d t d s \\
& =\int_{0}^{\infty} \int_{0}^{\infty} \min \left(\mu\left(\left\{f^{* \mu}>t\right\}\right), \mu\left(\left\{g^{* \mu}>s\right\}\right)\right) d t d s \\
& =\int_{0}^{\infty} \int_{0}^{\infty} \mu\left(\left\{f^{* \mu}>t\right\} \cap\left\{g^{* \mu}>s\right\}\right) d t d s=\int_{\mathbb{R}^{d}} f^{* \mu} g^{* \mu} d \mu
\end{aligned}
$$


where we used the fact that $\left\{f^{* \mu}>t\right\}$ and $\left\{g^{* \mu}>s\right\}$ are half-spaces of the form $\left\{\left(x_{1}, x^{\prime}\right) \in \mathbb{R} \times \mathbb{R}^{d-1}: x_{1}>r\right\}$ for some $r \in \mathbb{R}$ and so

$$
\min \left(\mu\left(\left\{f^{* \mu}>t\right\}\right), \mu\left(\left\{g^{* \mu}>s\right\}\right)\right)=\mu\left(\left\{f^{* \mu}>t\right\} \cap\left\{g^{* \mu}>s\right\}\right) .
$$

REMARK 2.2. Setting $g=\chi_{A}$ in Lemma 2.1 and thanks to (2.1) we get

$$
\int_{A} f d x \leq \int_{R_{A}^{\mu}} f^{* \mu}(x) d x=\int_{0}^{\mu(A)} f^{\star \mu}(s) d s .
$$

\section{A class of weighted isoperimetric inequalities}

Given a measurable function $V: \mathbb{R}^{d} \rightarrow \mathbb{R}$ we denote by $\mu[V]$ the absolutely continuous measure whose density equals $e^{V}$, that is, for any measurable set $E \subset \mathbb{R}^{d}$

$$
\mu[V](E)=\int_{E} e^{V(x)} d x
$$

in what follows with the scope of simplifying the notation, and if there is no risk of confusion, we will drop the dependence of $V$, writing $\mu$ instead of $\mu[V]$. Moreover we will often adopt the notation $x=\left(x_{1}, x^{\prime}\right) \in \mathbb{R} \times \mathbb{R}^{d-1}$ and denote by $R_{A}$ instead of $R_{A}^{\mu[V]}$ the right rearrangement of $A$ with respect to the measure $\mu[V]$. Given a Borel weight function $w: \mathbb{R} \rightarrow[0,+\infty]$ we define, for any open set $A$ with Lipschitz boundary, the following concept of weighted perimeter:

$$
P_{w, V}(A)=\int_{\partial A} w\left(x_{1}\right) e^{V(x)} d \mathcal{H}^{d-1}(x) .
$$

In the following proposition we show that, under suitable conditions on $w$ and $V$, the half-spaces of the form $\left\{\left(x_{1}, x^{\prime}\right): x_{1}>t\right\}$ are the only minimizers of the weighted perimeter among the sets of fixed volume with respect to the measure $\mu[V]$.

Proposition 3.1. Let $A \subset \mathbb{R}^{d}$ be a set with Lipschitz boundary. Suppose that $w: \mathbb{R} \rightarrow \mathbb{R}^{+}$and $V: \mathbb{R}^{d} \rightarrow \mathbb{R}$ are $C^{1}$-regular functions satisfying the following assumptions: 
(i) $\mu(A)=\mu\left(R_{A}\right)<+\infty$,

(ii) the function $\partial_{1} V(x)$ depends only on $x_{1}$ and $g(x):=-w^{\prime}\left(x_{1}\right)-$ $w\left(x_{1}\right) \partial_{1} V(x)$ is a non-negative decreasing function on the real line.

Then

$$
P_{w, V}(A) \geq P_{w, V}\left(R_{A}\right)
$$

Proof. We start by noticing that if $P_{w, V}(A)=+\infty$ there is nothing to prove. Hence we can suppose that

$$
P_{w, V}(A)<+\infty
$$

Let $e_{1}=(1,0, \ldots, 0) \in \mathbb{R}^{d}$ and consider the vector field $-e_{1} w\left(x_{1}\right) e^{V(x)}$. Its divergence is given by

$$
\operatorname{div}\left(-e_{1} w\left(x_{1}\right) e^{V}(x)\right)=\left(-w^{\prime}\left(x_{1}\right)-w\left(x_{1}\right) \partial_{1} V(x)\right) e^{V(x)}=g(x) e^{V(x)} .
$$

By an application of the Divergence Theorem we have

$$
\begin{aligned}
\int_{A} g(x) d \mu(x) & =\int_{A} \operatorname{div}\left(-e_{1} w\left(x_{1}\right) e^{V(x)}\right) d x \\
& =\int_{\partial A} w\left(x_{1}\right) e^{V(x)}\left\langle v_{A}(x),-e_{1}\right\rangle d \mathcal{H}^{d-1}(x) \\
& \leq \int_{\partial A} w\left(x_{1}\right) e^{V(x)} d \mathcal{H}^{d-1}(x)=P_{w, V}(A),
\end{aligned}
$$

where $v_{A}(x)$ is the outer unit normal to $\partial A$ at $x$. Let $t_{A}$ be a real number such that the right half-space $R_{A}=\left\{\left(x_{1}, x^{\prime}\right): x_{1} \geq t_{A}\right\}$ satisfies $\mu\left(R_{A}\right)=\mu(A)$. Then, since the outer normal of $R_{A}$ is the constant vector field $-e_{1}$, the inequality in (3.3) turns into an equality if we replace $A$ with $R_{A}$. Notice that by condition (ii) and (3.3) we have

$$
P_{w, V}\left(R_{A}\right)=\int_{R_{A} \backslash A} g d \mu+\int_{R_{A} \cap A} g d \mu \leq g\left(t_{A}\right) \mu(A)+P_{w, V}(A) .
$$

Thanks to assumption (i) and (3.2) such quantities are finite and so we get

$$
P_{w, V}(A)-P_{w, V}\left(R_{A}\right) \geq \int_{A} g(x) d \mu(x)-\int_{R_{A}} g(x) d \mu(x) .
$$

Since, by definition, $\mu(A)=\mu\left(R_{A}\right)<+\infty$ again by condition $(i)$ we obtain 
$\mu\left(A \backslash R_{A}\right)=\mu\left(R_{A} \backslash A\right)<+\infty$. Thus

$$
\begin{aligned}
\int_{A} g(x) & d \mu(x)-\int_{R_{A}} g(x) d \mu(x)=\int_{A \backslash R_{A}} g(x) d \mu(x)-\int_{R_{A} \backslash A} g(x) d \mu(x) \\
= & \int_{A \backslash R_{A}}\left(g(x)-g\left(t_{A} e_{1}\right)\right) d \mu(x)-\int_{R_{A} \backslash A}\left(g(x)-g\left(t_{A} e_{1}\right)\right) d \mu(x) .
\end{aligned}
$$

Since every $x \in A \backslash R_{A}$ (respectively $x \in R_{A} \backslash A$ ) satisfies $\left\langle x, e_{1}\right\rangle<t_{A}$ (respectively $\left\langle x, e_{1}\right\rangle>t_{A}$ ), by condition (ii) we deduce

$$
\begin{aligned}
P_{w, V}(A)-P_{w, V}\left(R_{A}\right) & \geq \int_{A \backslash R_{A}}\left|g(x)-g\left(t_{A} e_{1}\right)\right| d \mu(x)+\int_{R_{A} \backslash A}\left|g(x)-g\left(t_{A} e_{1}\right)\right| d \mu(x) \\
& =\int_{A \Delta R_{A}}\left|g(x)-g\left(t_{A} e_{1}\right)\right| d \mu \geq 0,
\end{aligned}
$$

where $A \Delta R_{A}=\left(A \backslash R_{A}\right) \cup\left(R_{A} \backslash A\right)$ stands for the symmetric difference between $A$ and $R_{A}$. This concludes the proof.

REMARK 3.2 (Necessity of the assumptions). We stress that the integrability condition $(i)$ is necessary to formulas (3.3) and (3.4) (and thus to our proof) to work.

Concerning condition (ii), we note that it is needed just for technical reasons. Nonetheless we stress that our proof offers a slightly stronger inequality than (3.1). Indeed the right-hand side of (3.5) may be seen as a modulus of continuity of the $L^{1}$ distance between $A$ and $R_{A}$. Thus it would be interesting to understand how much our hypotheses are far from optimality (compare also with [4, Remark 2.3]).

REMARK 3.3 (Equality cases). An inspection of the proof of Proposition 3.1, and in particular of inequality (3.3), shows that if $w>0$, then we have equality in (3.1) only if $A$ is equal to the half space $R_{A}$, up to set of zero $d$-dimensional Lebesgue measure. On the other hand, if the set $\{w=0\}$ has positive Lebesgue measure, we can not expect any kind of uniqueness for the equality cases of such an inequality.

EXAMPLE 3.4. A non-trivial example fulfilling condition (ii) of Proposition 3.1 is the following

$$
V\left(x_{1}, x^{\prime}\right)=-c\left(x_{1}\left|x_{1}\right|+\left|x^{\prime}\right|^{2}\right), \quad w\left(x_{1}\right)=e^{-a x_{1}},
$$


with $a, c>0$ constants satisfying $a^{2}-2 c \geq 0$. To prove this fact we initially observe that if $x_{1} \neq 0$ such a condition is equivalent to require that

$$
w^{\prime \prime}\left(x_{1}\right)+V_{1}^{\prime \prime}\left(x_{1}\right) w\left(x_{1}\right)+V_{1}^{\prime}\left(x_{1}\right) w^{\prime}\left(x_{1}\right) \geq 0
$$

which turns out to be equivalent, in our example, to

$$
a^{2}-2 c+2 a c\left|x_{1}\right| \geq 0 .
$$

Then, since $-w^{\prime}\left(x_{1}\right)-w\left(x_{1}\right) \partial_{1} V\left(x_{1}\right)$ is continuous in $x_{1}=0$, condition (ii) is satisfied everywhere.

To transform inequality (3.1) into a well posed isoperimetric problem, it would be more advisable to eliminate the integrability hypothesis $(i)$ in Proposition 3.1 by requiring that the measure $\mu\left(\mathbb{R}^{d}\right)<+\infty$. This fact, together with ordinary differential inequality required in assumption (ii), is seldom satisfied.

Hence, to get other instances of functions which fulfill inequality (3.6) together with the integrability property $(i)$ of Proposition 3.1 it is worth restricting our attention to the half-space

$$
\mathbb{R}_{+}^{d}=\left\{\left(x_{1}, x^{\prime}\right) \in \mathbb{R} \times \mathbb{R}^{d-1}: x_{1}>0\right\} .
$$

As an immediate corollary of Proposition 3.1 we get that the solution of the problem

$$
\min \left\{P_{w, V}(A): A \subseteq \mathbb{R}_{+}^{d}, \mu(A)=c, \partial A \text { Lipschitz }\right\}
$$

is given by $R_{c}=\left\{x_{1} \geq t_{c}\right\}$ where $t_{c}$ is such that $\mu\left(R_{c}\right)=c$.

REMARK 3.5. Notice that the non-mixed Gauss case, $w$ constant and $V(x)=-c|x|^{2}$, is not covered by our hypotheses. Nevertheless in this case examples of functions $w$ which satisfy the hypotheses of Proposition 3.1 are given by $w(t)=t^{-a}$ with $a \geq 1$ or $w(t)=b+e^{-a t}$, with $a, b \geq 0$ such that $a^{2}-2 c(1+b)>0$ (as can be easily seen reasoning as in the previous example). In the latter case at least if $b=0$ we have that

$$
w e^{V}=e^{a^{2} /(4 c)} \exp \left(-c\left|x+\mathbf{e}_{1} \frac{a}{2 c}\right|^{2}\right)
$$

where $\mathbf{e}_{1}=(1,0, \ldots, 0) \in \mathbb{R}^{d}$, which can be rephrased $\left({ }^{1}\right)$ as the fact that the

(1) As suggested us by an anonymous Referee. 
solutions of the isoperimetric problem in the half-space $\mathbb{R}_{+}^{d}$ with (suitable) mixed Gaussian conditions

$$
\min \left\{P_{\gamma_{\sigma, \eta}}(E): \gamma_{\sigma, 0}(E)=\text { constant, } E \subseteq \mathbb{R}_{+}^{d}, \partial E \text { Lipschitz }\right\}
$$

are right-half spaces. Here we denoted by $\gamma_{\sigma, \eta}$ the normal distribution whose covariance matrix is $\sigma \mathrm{Id}$ and whose mean vector $\eta$ is given by $\eta=-\frac{a}{2 c} \mathbf{e}_{1}$. We recall that, as pointed out in the Introduction, similar problems related to the Gauss measure are considered in [2], [6], [8], [9] and [13].

Notice that we defined the perimeter $P_{w, V}$ only for sets with Lipschitz boundary, but for our later applications it will be useful to have a definition of perimeter which comprehends also less regular subsets of $\mathbb{R}^{d}$. A measurable set $A$ is said to have locally finite (Euclidean) perimeter (we refer to [11] for a complete overview on the subject) if there exists a vectorvalued Radon measure $v_{A}$ called Gauss-Green measure of the set $A$ such that, for every $T \in C_{c}^{1}\left(\mathbb{R}^{d} ; \mathbb{R}^{d}\right)$, it holds true that

$$
\int_{A} \operatorname{div} T=\int_{\mathbb{R}^{d}}\left\langle T, d v_{A}\right\rangle .
$$

The perimeter of $A$ is defined in terms of the total variation of the GaussGreen measure of $A$ as $P(A)=\left|v_{A}\right|\left(\mathbb{R}^{d}\right)$. For any set $A$ of locally finite perimeter we then define the weighted perimeter $P_{w, V}$ by

$$
P_{w, V}(A)=w e^{V}\left|v_{A}\right|\left(\mathbb{R}^{d}\right) .
$$

Since when $A$ has Lipschitz boundary $\left|v_{A}\right|=\mathcal{H}^{d-1}\llcorner\partial A$, the above definition is coherent with the one given at the beginning of this section on such sets.

THEOREM 3.6. Let $w$ and $V$ non-negative and $C^{1}$-regular functions satisfying condition (ii) of Proposition 3.1. Suppose moreover that $\mu\left(\mathbb{R}_{+}^{d}\right)<+\infty$; then the problem

$$
\min \left\{P_{w, V}(A): A \subseteq \mathbb{R}_{+}^{d}, \mu(A)=c\right\}
$$

admits a solution, and this solution coincides with the one of (3.7).

Proof. Let $A$ be a measurable set of locally finite perimeter and suppose, by contraddiction, that $P_{w, V}(A)<P_{w, V}\left(R_{A}\right)$. We start by noticing that 
$P_{w, V}\left(R_{A}\right)<+\infty$, indeed, recalling (3.3) we have that

$$
P_{w, V}\left(R_{A}\right)=\int_{R_{A}} g(x) d \mu(x) \leq g(0) \mu(A) .
$$

By [11, Theorem II.2.8] we can find a sequence of sets $A_{n}$ with smooth boundary such that $\chi_{A_{n}} \rightarrow \chi_{A}$ in $L_{\mathrm{loc}}^{1}\left(\mathbb{R}^{d}\right)$ and $\left|v_{A_{n}}\right| \rightarrow^{*}\left|v_{A}\right|$, where $\rightarrow^{*}$ indicates the weak* convergence of Radon measures. Since $\mu\left(\mathbb{R}_{+}^{d}\right)<+\infty$, we also have that

$$
\chi_{A_{n}} \rightarrow \chi_{A} \text { in } L^{1}\left(\mathbb{R}^{d}, \mu\right)
$$

and, since $w e^{V}$ is a continuous function

$$
\lim _{n \rightarrow \infty} \int_{\mathbb{R}^{d}} w e^{V} d\left|v_{A_{n}}\right|=\int_{\mathbb{R}^{d}} w e^{V} d\left|v_{A}\right| .
$$

Thanks to (3.9) and Proposition 3.1 we get

$$
P_{w, V}(A)=\lim _{n \rightarrow \infty} P_{w, V}\left(A_{n}\right) \geq \lim _{n \rightarrow \infty} P_{w, V}\left(R_{A_{n}}\right) .
$$

We are left to show that $\lim _{n \rightarrow \infty} P_{w, V}\left(R_{A_{n}}\right)=P_{w, V}\left(R_{A}\right)$, but

$$
\left|P_{w, V}\left(R_{A}\right)-P_{w, V}\left(R_{A_{n}}\right)\right| \leq g(0)\left|\mu(A)-\mu\left(A_{n}\right)\right|,
$$

and we can conclude thanks to (3.8) and the fact that $\mu\left(\mathbb{R}_{+}^{d}\right)<+\infty$.

\section{Main result}

In this section we consider sets $E \subseteq \mathbb{R}_{+}^{d}$ and we define $d \mu=e^{V} d x$, $R_{E}=\left\{x_{1}>t_{E}\right\}$ where $t_{E} \in \mathbb{R}$ is such that $\mu\left(R_{E}\right)=\mu(E)$ and $f^{*}=f^{* \mu}$ the right rearrangement of a function $f$ with respect to $\mu$. In what follows we consider problems of the form

$$
\begin{cases}-\operatorname{div}\left(w^{2} e^{V} \nabla u\right)=f e^{V} & \text { in } E \\ u=0 & \text { on } \partial E\end{cases}
$$

which must be intended in weak sense. Precisely, a solution of (4.1) is a function $u \in H_{0}^{1}\left(e^{V}, w^{2} e^{V}, E\right)$, defined as the closure of $C_{0}^{\infty}(E)$ with respect to the norm

$$
\|u\|_{H_{0}^{1}\left(e^{V}, w^{2} e^{V}, E\right)}=\left(\int_{E} u^{2} e^{V} d x+\int_{E}|\nabla u|^{2} w e^{V} d x\right)^{1 / 2},
$$


and which satisfies

$$
\int_{E}\langle\nabla u, \nabla \phi\rangle w^{2} e^{V} d x=\int_{E} f \phi e^{V} d x
$$

for any $\phi \in H_{0}^{1}\left(e^{V}, w^{2} e^{V}, E\right)$.

The main scope of this section is to prove a priori estimates for the solutions of problem (4.1). For this reason we shall always consider that a solution $u$ exists. Clearly this requirement depends on the choice of $w, V$ and $f$. General instances of such functions for which the existence of a solution for problem (4.1) is guaranteed, can be found in [14] (see also [2], [8], [9], and [13]). Here we limit ourselves to state that most of the examples considered in Remark 3.5, as the mixed-Gaussian case $V(x)=-c|x|^{2}$, $w(t)=b+e^{-a t}$ with $a^{2}-2 c(1+b)>0$ and $b$ strictly positive, are covered by the cases considered in [14], whenever $f \in L^{2}\left(E, e^{V}\right)$.

MaIn Theorem. Suppose that the set $E \subset \mathbb{R}_{+}^{d}=\left\{\left(x_{1}, x^{\prime}\right): x_{1}>0\right\}$ and the functions $w:[0,+\infty] \rightarrow(0,+\infty]$ and $V: \mathbb{R}^{d} \rightarrow \mathbb{R}$ satisfy the hypotheses of Proposition 3.1. Consider the two problems

$$
\begin{cases}-\operatorname{div}\left(w^{2} e^{V} \nabla u\right)=f e^{V} & \text { in } E \\ u=0 & \text { on } \partial E\end{cases}
$$

and

$$
\begin{cases}-\operatorname{div}\left(w^{2} e^{V} \nabla v\right)=f^{*} e^{V} & \text { in } R_{E} \\ v=0 & \text { on } \partial R_{E}\end{cases}
$$

where $0<f \in L^{2}\left(\mathbb{R}_{+}^{d}, \mu\right)$. Then the problem (4.4) has as solution the one variable function $v(z)$ given by

$$
v\left(\left(z, z^{\prime}\right)\right)=v(z)=\int_{\mu\left(\left\{x_{1} \geq z\right\}\right)}^{\mu\left(R_{E}\right)} \frac{1}{h^{2}(s)}\left(\int_{0}^{s} f^{*}(\xi) d \xi\right) d s
$$

where

$$
h(m)=w\left(\Phi^{-1}(m)\right) \int_{\mathbb{R}^{d-1}} \mu\left(\Phi^{-1}(m), x^{\prime}\right) d x^{\prime}
$$

being $\Phi(t)=\mu\left(\left\{x_{1}>t\right\}\right)$. Moreover, for any solution $u$ of the problem (4.3), 
we have

$$
u^{*}(x) \leq v(x),
$$

and, for any $q \in(0,2]$,

$$
\int_{E}|\nabla u|^{q} w^{q} d \mu \leq \int_{R_{E}}|\nabla v|^{q} w^{q} d \mu
$$

Proof. Let us suppose for the moment that the function $v$ given in (4.5) is a solution for the problem (4.4). To prove (4.7) and (4.8) we consider the functions $\phi_{h}$ defined as

$$
\phi_{h}(x)= \begin{cases}\operatorname{sign}(u) & \text { if }|u|>t+h \\ \frac{u(x)-t \operatorname{sign} u(x)}{h} & \text { if }|u| \in[t, t+h) \\ 0 & \text { if }|u|<t,\end{cases}
$$

where $0 \leq t<\operatorname{ess} \sup |\mathrm{u}|$ and $h>0$. Notice that, for every $h>0$, $\phi_{h}$ is an admissible test function, since the solution $u$ belongs to the space $H_{0}^{1}\left(e^{V}, w^{2} e^{V}, E\right)$. Then (4.2) turns into

$$
\frac{1}{h} \int_{\{|u| \in[t, t+h)\}}\langle\nabla u, \nabla u\rangle w^{2} d \mu=\frac{1}{h} \int_{\{|u| \in[t, t+h)\}} f\left(u-t \frac{u}{|u|}\right) d \mu+\int_{\{|u|>t+h\}} f \operatorname{sign}(u) d \mu .
$$

Taking the limit for $h \rightarrow 0$, we get

$$
-\frac{d}{d t} \int_{\{|u|>t\}}|\nabla u|^{2} w^{2} d \mu=\int_{\{|u|>t\}} f d \mu .
$$

Let us analyze the left-hand side of equation (4.9). We claim that the following inequality holds true for almost every $t$ :

$$
-\frac{d}{d t} \int_{\{|u|>t\}}|\nabla u|^{2} w^{2} d \mu \geq \frac{\left(-\frac{d}{d t} \int_{\{|u|>t\}}|\nabla u| w d \mu\right)^{2}}{-\mu_{u}^{\prime}(t)},
$$

where $\mu_{u}(t)$ is the distribution function of $u$ introduced in the Section 2 . Indeed $\mu_{u}(t)$ is a decreasing function and thence it is derivable for almost every $t$, thanks to the Hölder inequality we get 


$$
\begin{aligned}
-\frac{d}{d t} \int_{\{|u|>t\}} \mid & \nabla u\left|w d \mu=\lim _{h \rightarrow 0} \frac{1}{h} \int_{t<|u|<t+h}\right| \nabla u \mid w d \mu \\
& \leq \lim _{h \rightarrow 0}\left(\int_{\{t<|u|<t+h\}}|\nabla u|^{2} w^{2} d \mu\right)^{1 / 2}\left(\int_{\{t<|u|<t+h\}} \frac{1}{h^{2}} d \mu\right)^{1 / 2} \\
& =\lim _{h \rightarrow 0}\left(\frac{1}{h} \int_{\{t<|u|<t+h\}}|\nabla u|^{2} w^{2} d \mu\right)^{1 / 2}\left(\frac{1}{h} \int_{\{t<|u|<t+h\}} 1 d \mu\right)^{1 / 2} \\
& =\left(-\frac{d}{d t} \int_{\{|u|>t\}}|\nabla u|^{2} w^{2} d \mu\right)^{1 / 2}\left(-\mu_{u}^{\prime}(t)\right)^{1 / 2}
\end{aligned}
$$

By the Co-Area formula and the fact that $w$ is strictly positive and $C^{1}$ regular, we easily get that the set $\{u>t\}$ is a set of locally finite (Euclidean) perimeter. Thus, thanks to Proposition 3.1 and Theorem 3.6 we get

$$
-\frac{d}{d t} \int_{\{|u|>t\}}|\nabla u| w d \mu=\int_{\{|u|=t\}} w d \mu=P_{w, V}(\{|u|>t\}) \geq P_{w, V}\left(\left\{u^{*}>t\right\}\right) .
$$

We introduce the function

$$
\Phi(t)=\mu\left(\left\{x_{1}>t\right\}\right) .
$$

We recall that the weight function $w$ is constant on the boundary of the super level sets of $u^{*}$, so that the perimeter of $\left\{u^{*}>t\right\}$ can be written as

$$
P_{w, V}\left(\left\{u^{*}>t\right\}\right)=w(\tau) \int_{\mathbb{R}^{d-1}} \mu\left(\tau, x^{\prime}\right) d x^{\prime}
$$

Moreover $\tau \in \mathbb{R}$ satisfies $\mu_{u^{*}}(t)=\Phi(\tau)$ that is $\tau=\Phi^{-1}\left(\mu_{u^{*}}(t)\right.$ ) (notice that $\Phi$ is a strictly decreasing function and thus invertible) so that we can write the previous formula as

$$
P_{w, V}\left(\left\{u^{*}>t\right\}\right)=w\left(\Phi^{-1}\left(\mu_{u^{*}}(t)\right)\right) \int_{\mathbb{R}^{d-1}} \mu\left(\Phi^{-1}\left(\mu_{u^{*}}(t)\right), x^{\prime}\right) d x^{\prime}:=h\left(\mu_{u^{*}}(t)\right) .
$$


Plugging (4.12) in (4.10), and recalling (4.13) we get that

$$
-\frac{d}{d t} \int_{\{|u|>t\}}|\nabla u|^{2} w^{2} d \mu \geq \frac{h\left(\mu_{u^{*}}(t)\right)^{2}}{-\mu_{u^{*}}^{\prime}(t)} .
$$

We pass now to estimate the right-hand side of (4.9): equation (2.2) with $A=\{|u|>t\}$ turns into

$$
\int_{\{|u|>t\}} f d \mu \leq \int_{\left\{\left|u^{*}\right|>t\right\}} f^{*} d \mu=\int_{0}^{\mu_{u^{*}}(t)} f^{\star}(s) d s .
$$

Combining (4.15) and (4.14) we get

$$
\frac{\left(\int_{0}^{\mu_{u^{*}}(t)} f^{\star}(s) d s\right) \mu_{u^{*}}^{\prime}(t)}{h^{2}\left(\mu_{u^{*}}(t)\right)} \leq-1 .
$$

Reasoning analogously for the function $v$, we easily see that, since $v$ is constant on every set $\left\{x_{1}=t\right\}$ and since $v=v^{*}$, (4.16) holds for $v$ as an equality. Consider now the real function

$$
F(r)=\frac{\int_{0}^{r} f(s) d s}{h(r)^{2}},
$$

and let $G$ be a primitive of $F$. Since $F \geq 0$, we have that $G$ is increasing. Moreover by our previous analysis we have that

$$
F\left(\mu_{u^{*}}(t)\right) \mu_{u^{*}}^{\prime}(t) \leq-1=F\left(\mu_{v}(t)\right) \mu_{v}^{\prime}(t) .
$$

We recall that here $\mu_{u^{*}}^{\prime}(t)$ denotes the derivative almost everywhere of the function $\mu_{u^{*}}(t)$. Moreover $t \mapsto G\left(\mu_{u^{*}}(t)\right)$ is a monotone non-increasing function which satisfies the chain rule in any point of differentiability of $\mu_{u^{*}}$, so that, by [1, Corollary 3.29], we get that

$$
G\left(\mu_{u^{*}}(t)\right) \leq G\left(\mu_{u^{*}}(0)\right)+\int_{0}^{t} F\left(\mu_{u^{*}}(\tau)\right) \mu_{u^{*}}^{\prime}(\tau) d \tau .
$$

On the other hand, being $\mu_{v}(t)$ an absolutely continuous function (since $v$ is a $C^{1}$-regular with positive derivative one variable function) we have

$$
G\left(\mu_{v}(t)\right)=G\left(\mu_{u^{*}}(0)\right)+\int_{0}^{t} F\left(\mu_{v}(\tau)\right) \mu_{v}^{\prime}(\tau) d \tau,
$$


so that, since $G\left(\mu_{v}(0)\right)=G\left(\mu_{u^{*}}(0)\right)$, we get that $G\left(\mu_{u^{*}}(t)\right) \leq G\left(\mu_{v}(t)\right)$. This implies that $\mu_{u^{*}}(t) \leq \mu_{v}(t)$ for any $t$ and hence that $u^{*} \leq v$, since $u^{*}$ and $v$ depends only on $x_{1}$ and are increasing functions of such a variable.

We pass now to the proof of (4.8). Using the Hölder inequality and reasoning as before we obtain, for $0<q \leq 2$,

$$
\begin{aligned}
-\frac{d}{d t} \int_{\{|u|>t\}}|\nabla u|^{q} w^{q} d \mu=\lim _{h \rightarrow 0} \frac{1}{h} \int_{\{t<|u|<t+h\}}|\nabla u|^{q} w^{q} d \mu \\
\leq \lim _{h \rightarrow 0}\left(\frac{1}{h} \int_{\{t<|u|<t+h\}}|\nabla u|^{2} w^{2} d \mu\right)^{q / 2}\left(\frac{1}{h} \int_{\{t<|u|<t+h\}} d \mu\right)^{1-q / 2} \\
=\left(-\frac{d}{d t} \int_{\{|u|>t\}}|\nabla u|^{2} w^{2} d \mu\right)^{q / 2}\left(-\mu_{u}^{\prime}(t)\right)^{1-q / 2}
\end{aligned}
$$

Recalling (4.9) and (4.15) we have

$$
-\frac{d}{d t} \int_{\{|u|>t\}}|\nabla u|^{2} w^{2} d \mu \leq \int_{0}^{\mu_{u^{*}}(t)} f^{*}(s) d s
$$

thus

$$
-\frac{d}{d t} \int_{\{|u|>t\}}|\nabla u|^{q} w^{q} d \mu \leq\left(\int_{0}^{\mu_{u^{\star}}(t)} f^{\star}(s) d s\right)^{q / 2}\left(-\mu_{u}^{\prime}(t)\right)^{1-q / 2} .
$$

Combining (4.19) and (4.16) we finally get

$$
-\frac{d}{d t} \int_{\{|u|>t\}}|\nabla u|^{q} w^{q} d \mu \leq\left(-\mu_{u^{*}}^{\prime}(t)\right)\left(h\left(\mu_{u^{*}}(t)\right)^{-1} \int_{0}^{\mu_{u^{*}}(t)} f^{\star}(s) d s\right)^{q} .
$$

By integrating on both side between 0 and $+\infty$, we get

$$
\int_{E}|\nabla u|^{q} w^{q} d \mu \leq \int_{0}^{\infty}\left(-\mu_{u^{*}}^{\prime}(t)\right)\left(h\left(\mu_{u^{*}}(t)\right)^{-1} \int_{0}^{\mu_{u^{*}}(t)} f^{\star}(s) d s\right)^{q} d t .
$$


We perform the change of variables $r=\mu_{u^{*}}(t)$, so that the above equation turns into

$$
\int_{E}|\nabla u|^{q} w^{q} d \mu \leq \int_{0}^{\mu(E)}\left(h(r)^{-1} \int_{0}^{r} f^{\star}(s) d s\right)^{q} d r .
$$

By a straightforward inspection of those steps we notice that $v$ satisfies

$$
\int_{R_{E}}|\nabla v|^{q} w^{q} d \mu=\int_{0}^{\infty}\left(-\mu_{v}^{\prime}(t)\right)\left(h\left(\mu_{v}(t)\right)^{-1} \int_{0}^{\mu_{v}(t)} f^{\star}(s) d s\right)^{q} d t ;
$$

By performing the change of variables $r=\mu_{v}(t)$ we find

$$
\int_{R_{E}}|\nabla v|^{q} w^{q} d \mu=\int_{0}^{\mu\left(R_{E}\right)}\left(h(r)^{-1} \int_{0}^{r} f^{\star}(s) d s\right)^{q} d r .
$$

Since $\mu(E)=\mu\left(R_{E}\right)$ we get the desired result.

We are left to prove that the function $v$ given by (4.5) is a solution of problem (4.4). We start by noticing that equation (4.16) suggests how to derive (4.5): indeed, as we pointed out, any solution $v$ of (4.4) such that $v=v^{*}$ satisfies

$$
\frac{\int_{0}^{\mu_{v}(t)} f^{\star}(s) d s}{h^{2}\left(\mu_{v}(t)\right)} \mu_{v}^{\prime}(t)=-1 .
$$

By integrating both sides between 0 and $r$ we obtain

$$
\int_{0}^{r} \frac{\int_{0}^{\mu_{v}(t)} f^{\star}(s) d s}{h^{2}\left(\mu_{v}(t)\right)} \mu_{v}^{\prime}(t) d t=-r
$$

so that, by performing the change of variables $m=\mu_{v}(t)$, we get

$$
\int_{\mu_{v}(r)}^{\mu\left(R_{E}\right)} \frac{\int_{0}^{m} f^{\star}(s) d s}{h^{2}(m)} d m=r
$$


which is equivalent to

$$
v\left(z, z^{\prime}\right)=\int_{\mu\left\{x_{1}>z\right\}}^{\mu\left(R_{E}\right)} \frac{\int_{0}^{m} f^{\star}(s) d s}{h^{2}(m)} d m,
$$

that is (4.5). Notice that $v$ is strictly decreasing and belongs to $C_{\text {loc }}^{1,1}\left(R_{E}\right)$. Indeed, recalling (4.6) one can explicitly compute

$$
\nabla v\left(z, z^{\prime}\right)=e_{1} \frac{\partial v}{\partial z}\left(z, z^{\prime}\right)=-e_{1} \frac{\int_{0}^{\mu\left\{x_{1}>z\right\}} f^{\star}(s) d s}{w^{2}(z) \int_{R^{d-1}} e^{V\left(z, x^{\prime}\right)} d x^{\prime}},
$$

where $e_{1}=(1,0, \ldots, 0) \in \mathbb{R}^{d}$. Since $f^{\star}$ is a decreasing and locally integrable function, then $f^{\star} \in L_{\mathrm{loc}}^{\infty}(\mathbb{R})$; thus, being $z \mapsto \mu\left(\left\{x_{1}>z\right\}\right) C^{1}$-regular, we get that $\int_{0}^{\mu\left\{x_{1}>z\right\}} f^{\star}(s) d s$ is a locally Lipschitz function. Moreover the denominator is locally Lipschitz as well, and locally bounded away from zero. Hence we have that $\nabla v$ is locally Lipschitz. Thus, recalling that $\partial_{1} V$ depends only on the first variable $x_{1}$ it is possible to explicitly compute the divergence of $w^{2} \nabla v e^{V}$ and check that it satisfies (4.4). This concludes the proof of the theorem.

Acknowledgments. The authors thank L. Brasco and G. De Philippis for useful discussions on the topic. They are also grateful to the anonymous referee for several suggestions and remarks.

\section{REFERENCES}

[1] L. Ambrosio, N. Fusco, D. Pallara: Functions of bounded variation and free discontinuity problems, Oxford Mathematical Monographs, The Clarendon Press Oxford University Press, New York, 2000.

[2] M.F. Betta, F. Brock, A. Mercaldo, M. R. Posteraro: A comparison result related to Gauss measure, C. R. Math. Acad. Sci. Paris, 334 (2002), 451-456.

[3] M.F. Betta, F. Brock, A. Mercaldo, M.R. Posteraro, A weighted isoperimetric inequality and applications to symmetrization, J. lnequal. Appl., 4 (1999), 215-240.

[4] L. Brasco, G. De Philippis, B. Ruffini: Spectral optimization for the Stekloff-Laplacian: the stability issue, J. Funct. Anal., 262 (2012), 4675-4710.

[5] L. Brasco, G. Franzina: An anisotropic eigenvalue problem of Stekloff type 
and weighted Wulff inequalities, Nonlinear Differential Equations Appl., 20 (2013), 1795-1830.

[6] F. Brock, F. Chiacchio, A. Mercaldo: A class of degenerate elliptic equations and a Dido's problem with respect to a measure, J. Math. Anal. Appl., 348 (2008), 356-365.

[7] F. Brock, F. Chiacchio, A. Mercaldo: Weighted isoperimetric inequalities in cones and applications, Nonlinear Anal., 75 (2012), 5737-5755.

[8] G. Di Blasio: Linear elliptic equations and Gauss measure, J. Inequal. Pure Appl. Math, 4 (2003), 1-11.

[9] G. Di Blasio, F. Feo, M. R. Posteraro: Regularity results for degenerate elliptic equations related to Gauss measure, Math. Inequal. Appl., 10 (2007), 771-797.

[10] E.H. Lieb, M. Loss: Analysis, American Mathematical Society, 1997.

[11] F. MAGGI: Sets of finite perimeter and geometric variational problems, Cambridge Studies in Advanced Mathematics, 2012.

[12] G. TALEnTI: Elliptic equations and rearrangements, Ann. Scuola Norm. Sup. Pisa Cl. Sci., 4 (1976), 697-718.

[13] Y. J. Tian, F. Q. Li: On the Case of Equalities in Comparison Results for Elliptic Equations Related to Gauss Measure, J. Math. Res. Exposition, 30 (2010), 761-774.

[14] N. S. TRUDINGER: Linear elliptic operators with measurable coefficients, Ann. Scuola Norm. Sup. Pisa Cl. Sci., 27 (1973), 265-308.

Manoscritto pervenuto in redazione il 16 dicembre 2013. 